\title{
Theoretical Study of Conformational Disorder and Selective Adsorption of Small Organic Molecules in the Flexible Metal-Organic Framework Material MIL-53-Fe
}

\author{
Sanliang Ling, ${ }^{\mathrm{a}^{*}}$ Richard I. Walton, ${ }^{\mathrm{b}}$ and Ben Slater ${ }^{\mathrm{a}}$ \\ ${ }^{a}$ Department of Chemistry, University College London, 20 Gordon Street, London, WC1H 0AJ, United \\ Kingdom \\ ${ }^{\mathrm{b}}$ Department of Chemistry, University of Warwick, Coventry, CV4 7AL, United Kingdom \\ E-mails: s.ling@ucl.ac.uk; b.slater@ucl.ac.uk
}

\begin{abstract}
Many of the potential applications for metal-organic framework (MOF) materials require molecular level understanding of their adsorption of small organic molecules, which are not readily accessible from experiment. Through high-level van der Waals corrected, hybrid density functional theory calculations, we elucidate the adsorption configurations of several, representative small organic guest molecules in an archetypal flexible MOF material, MIL-53-Fe. The predicted relative energies between low-energy adsorption configurations of 1,4-benzoquinone in MIL-53-Fe are in very good agreement with the thermal transition temperatures observed experimentally and suggest thermodynamic factors govern the precise arrangements and loading of guests in the MOF host. Experimentally observed conformational disorder of small organic molecules in MIL-53-Fe is explained by predicted multiple low-energy adsorption configurations that are comparable with the thermal energy of the guests, $k T$. Finally, we show that the previously observed selective adsorption of pyridine and 2,6-lutidine molecules over water by MIL-53-Fe, can be rationalised through a careful analysis of the hostguest and guest-guest interactions and is controlled by thermodynamic factors.
\end{abstract}

\section{Introduction}

Metal-organic framework (MOF) materials have been studied extensively for a range of potential applications, including molecular sieving, ${ }^{1}$ gas storage, ${ }^{2-4}$ gas sensors,${ }^{5}$ drug delivery, ${ }^{6}$ lithium-ion battery electrodes $^{7}$ and heterogeneous catalysis. ${ }^{8}$ While significant progress has been made in the rational design of new MOF materials with various topologies and chemical compositions in recent years, ${ }^{9-10}$ generally, there is still a lack of molecular level information on the mechanisms of adsorption and sieving. Experimentally, highresolution powder X-ray diffraction (XRD), ${ }^{11}$ neutron powder diffraction ${ }^{12}$ and X-ray absorption fine structure spectroscopy ${ }^{13}$ offer the potential to resolve the position of the guest in the host and the nature of their chemical interactions. However, due to the softness of some MOF materials, it is rather difficult to fully resolve the adsorption pattern of organic molecules inside a MOF. The situation becomes even more complicated when a MOF material swells or contracts under the influence of different guest molecules inside the pore, ${ }^{11,14-15}$ i.e. the so-called breathing effect. The archetypal example of such a MOF material is MIL-53, ${ }^{16}$ of the general formula $[\mathrm{M}(\mathrm{OH})(\mathrm{BDC})]$, where $\mathrm{M}=\mathrm{Al}, \mathrm{Ga}$, In, $\mathrm{Sc}, \mathrm{V}(\mathrm{III}), \mathrm{Cr}(\mathrm{III})$ or $\mathrm{Fe}(\mathrm{III})$, and $\mathrm{BDC}^{2-}=1,4-$ benzenedicarboxylate. In a recent study, MIL-53-Fe was loaded with different organic guest molecules by Millange et al. ${ }^{11}$ and a change in the cell volume was found that was only weakly correlated with the size of the guest, implying that the cell volumes are determined by specific, anisotropic host-guest interactions and guest-guest interactions, rather than a simple isotropic, radially dependent interaction. In another work by the same authors, it was found that while hydrated MIL-53-Fe adsorbed both pyridine and 2,6-lutidine (2,6dimethylpyridine) initially, it selectively adsorbed 2,6-lutidine over pyridine with time. ${ }^{17}$ This example illustrates the importance of gaining a molecular level understanding of the interactions between a MOF material and adsorbed organic molecules. In this work, by using state-of-the-art density functional theory (DFT) methods, we study the adsorption of a sub-set of the organic molecules studied by Millange et al., ${ }^{11}$ including quinone (1,4-benzoquinone), pyridine, and 2,6-lutidine (shown in Figure 1), in the MIL-53-Fe structure (depicted in Figure 2). By comparing simulation with experiment, we identify the detailed nature of the 
interactions between these small organic molecules and MIL-53-Fe, provide explanations for the observed guest configurations and rationalize why the guest configurations change in response to temperature.

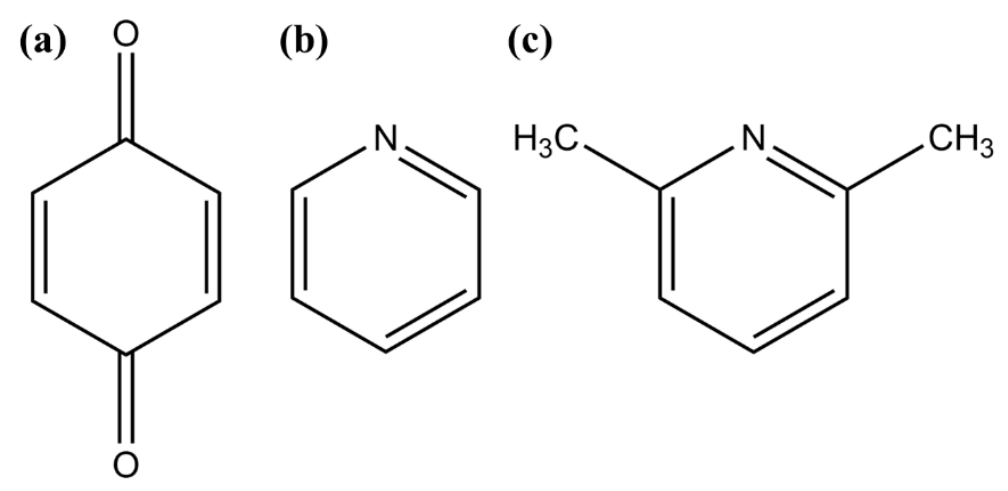

Figure 1: Structures of the three guest molecules considered (a) 1,4-benzoquinone, (b) pyridine, and (c) 2,6lutidine.
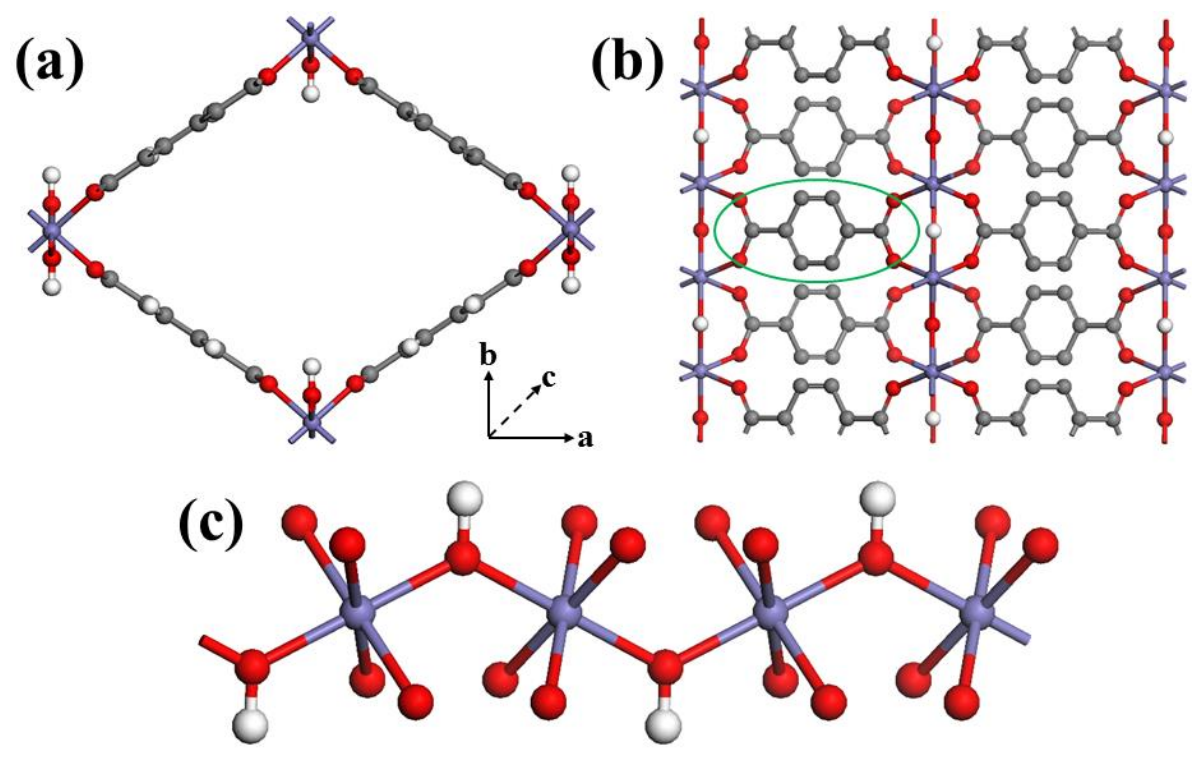

Figure 2: Structure of fully expanded MIL-53-Fe and its building blocks, (a) view along lattice vector $c$ showing the one-dimensional channel, (b) view in the ac plane showing the inorganic chain and $\mathrm{BDC}^{2-}$ organic linker (highlighted in green circle, hydrogen atoms belong to $\mathrm{BDC}^{2-}$ are omitted for clarity), and (c) inorganic chain showing $\mathrm{FeO}_{4}(\mathrm{OH})_{2}$ octahedra. Red: oxygen; grey: carbon; mauve: iron; white: hydrogen.

The adsorption of small organic molecules by MOF materials is dominated by two types of interactions: host-guest and guest-guest interactions. ${ }^{18}$ With respect to the adsorption of quinone, pyridine, and 2,6-lutidine in MIL-53-Fe, $\mathrm{BDC}^{2-}$ is the organic linker and $\mathrm{OH}^{-}$is the inorganic linker (Figure 2), and it is these two functional groups which modulate the host-guest interactions. We note that $\sim 20 \%$ of $\mathrm{OH}^{-}$inorganic linkers in the experimental sample of MIL-53-Fe were occupied by isovalent $\mathrm{F}^{-}$ions. ${ }^{17}$ As a first order approximation, all models of MIL-53-Fe contain only $\mathrm{OH}^{-}$inorganic linkers; we assume that because the $\mathrm{F}^{-}$ions are likely to be disordered and are minority species, the influence of guest-hydroxide interactions is likely to control the observed response of the lattice. Hence possible host-guest interactions include: (1) $\pi-\pi$, (2) CH- $\pi$, and (3) hydrogen bonds. Guest-guest interactions are dominated by $\pi$ - $\pi$ stacking, and hydrogen bonds may also play some role, depending on the particular orientations of guests inside the pore. A more detailed summary of the possible host-guest and guest-guest interactions between MIL-53-Fe and the three different organic molecules are shown in Table 1. It should be noted that the strengths of these interactions are different, and generally speaking, the strengths follow the order of hydrogen bonds $>\pi-\pi>\approx \mathrm{CH}-\pi$, and for hydrogen bonds, $\mathrm{N} \cdots \mathrm{HO}$ bonds are usually stronger than $\mathrm{O} \cdots \mathrm{HO}$. The final adsorption configuration of the guest molecules inside the pore of the host is determined by a subtle balance of these different interactions (host-guest, guest-guest and 
hydrogen bonds, $\pi-\pi, \mathrm{CH}-\pi)$, including guest-guest repulsion as well as attraction but here we focus on stabilising interactions.

\begin{tabular}{lll}
\hline Guest & Host-Guest & Guest-Guest \\
\hline quinone & $\pi(\mathbf{G})-\pi(\mathbf{H})$ & $\pi-\pi$ \\
& $\mathrm{CH}(\mathbf{G})-\pi(\mathbf{H})$ & $\mathrm{O} \cdots \mathrm{HC}$ \\
& $\mathrm{O}(\mathbf{G}) \cdots \mathrm{HO}(\mathbf{H})$ & \\
\hline pyridine & $\pi(\mathbf{G})-\pi(\mathbf{H})$ & $\pi-\pi$ \\
& $\mathrm{CH}(\mathbf{G})-\pi(\mathbf{H})$ & \\
& $\mathrm{N}(\mathbf{G}) \cdots \mathrm{HO}(\mathbf{H})$ & \\
\hline 2,6-lutidine & $\pi(\mathbf{G})-\pi(\mathbf{H})$ & $\pi-\pi$ \\
& $\mathrm{CH}(\mathbf{G})-\pi(\mathbf{H})$ & \\
& $\mathrm{N}(\mathbf{G}) \cdots \mathrm{HO}(\mathbf{H})$ & \\
\hline
\end{tabular}

Table 1: Possible host-guest and guest-guest interactions between MIL-53-Fe and quinone, pyridine and 2,6lutidine. Capital letters in brackets indicates the moiety either belongs to the host $(\mathbf{H})$ or the guest $(\mathbf{G})$.

Hydrogen bonds are indicated by “...", and other interactions are indicated by ".".

Simulations of the adsorption of sorbates in MOF materials often consider an idealised system at $0 \mathrm{~K}$, neglecting thermal effects and with a fixed loading in the MOF. Frequently in experiments, it can be difficult to eliminate all solvent and so multiple guests may be present within the MOF. Solvent molecules themselves can act a guests, reducing the intended guest loading from its hypothetical limit at any given state point. ${ }^{19}$ These factors affect the configurations of small organic molecules adopted in the host, and consequently the predicted ground state adsorption configuration can deviate from what is actually observed in the experiment. In the case of single component adsorption quinone and pyridine in MIL-53-Fe, for example, for quinone, two ordered phases were identified at two different temperatures, ${ }^{20}$ and for pyridine, one ordered phase was identified at room temperature, and there was evidence of another phase between $363 \sim 423 \mathrm{~K}$ which could not be indexed due to poor crystallinity. ${ }^{17}$ To account for these metastable adsorption phases, which cannot be easily identified in experiments but are still important in relation to the applications being considered for MOF materials, in addition to the ground state adsorption configuration which has already been solved by experiment for each adsorbate molecule, we also consider several other possible adsorption configurations.

As already stated, solvent molecules can affect the uptake and adsorption positions of guest molecules in MOFs. This is observed for the adsorption of 2,6-lutidine in MIL-53-Fe. ${ }^{17}$ Unlike the case of pyridine, where pyridine molecules displace occluded water molecules in MIL-53-Fe, ${ }^{17}$ upon exposure of MIL-53-Fe[ $\left.\mathrm{H}_{2} \mathrm{O}\right]$ to 2,6-lutidine, the water molecules remain in the channel and act as a bridge between MIL-53-Fe and 2,6lutidine. ${ }^{17}$ Millange et al. concluded this was due to the presence of methyl groups in 2,6-lutidine (see Figure 1) which prevents direct hydrogen bonding between MIL-53-Fe and 2,6-lutidine. ${ }^{17}$ It remains unclear why the same situation did not occur in the adsorption of pyridine molecules in MIL-53-Fe. In this study, we seek to explain this observation by performing high-level atomistic, density functional theory simulations. These simulations will help us to better understand the competition between guest-guest interactions and host-guest interactions in MOFs, as well as the role of temperature and solvent molecules in the modulation of these interactions.

Simulations offer an additional means of investigating structures and examining the balance between electrostatic and van der Waals forces that determine the position and strength of guest adsorption in MOFs. Indeed, with recent developments in van der Waals (vdW) corrected DFT methods, ${ }^{21}$ good agreement between theory and experiment can often be obtained for MOFs of varied density, ${ }^{22-24}$ which demonstrates the efficiency and reliability of vdW corrected DFT methods for describing both structural and energetic properties of MOFs. For example, Düren and co-workers have shown that ab initio molecular dynamics simulations based on vdW corrected DFT method can be used to elucidate the breathing behaviour of MIL-53-Sc upon temperature variation and $\mathrm{CO}_{2}$ loading. ${ }^{25}$ This prompts us to employ vdW corrected DFT methods, rather than empirical force fields which were frequently used in previous studies of MOFs, ${ }^{25-28}$ to study the interactions between small organic molecules and MIL-53-Fe.

\section{Methods}


All calculations were performed using the $\mathrm{CP} 2 \mathrm{~K}$ code which uses a mixed Gaussian/plane-wave basis set. ${ }^{29-30}$ In the current study, we have employed triple- $\zeta$ polarization quality (TZVP) Gaussian basis sets and a 800 Ry plane-wave cutoff for the auxiliary grid, in conjunction with the Goedecker-Teter-Hutter pseudopotentials. ${ }^{31-32} \mathrm{~A}$ TZVP basis set is particularly important to minimize the basis set superposition error (BSSE) and to correctly describe some of the non-bonding interactions between host and guest and between different guest molecules, in terms of both binding distances and binding energies. Both of the latter effects are important to produce a good description to the lattice parameters of MIL-53 type materials in the presence or absence of guest molecules. ${ }^{24}$ The counterpoise method ${ }^{33}$ was used to correct for BSSEs in all binding energy calculations. The hybrid density functional theory (DFT) method, HSE06, ${ }^{34}$ with $25 \%$ short-range Hartree-Fock exchange (HFX), together with Grimme's D3 van der Waals correction, ${ }^{35}$ including the Axilrod-Teller-Muto three-body terms, ${ }^{36}$ was used for all total energy calculations and structural optimizations, including lattice parameters and atomic positions. The HFX calculations, which are part of HSE06, were performed using the auxiliary density matrix method (ADMM) ${ }^{37}$ The ADMM approach projects the expensive HFX calculations with the density matrix from a large primary basis set onto a much smaller and rapidly decaying auxiliary density matrix from a smaller auxiliary basis set, which significantly accelerates the HFX calculations and reduces the computational cost. It enables us to consider relatively large systems (with the largest system containing 576 atoms) at hybrid DFT level at a relatively modest computational cost. The hybrid DFT approach is necessary here to provide a reasonable description of the electronic structures, band gaps and the magnetic interactions of high-spin $\mathrm{Fe}^{3+}$ cations, which contain unpaired electrons $\left(3 \mathrm{~d}^{5}\right.$, i.e. $\mathrm{t}_{2 \mathrm{~g}}{ }^{3} \mathrm{eg}_{\mathrm{g}}{ }^{2}$ ). Spin polarised calculations were performed for all calculations involving $\mathrm{Fe}^{3+}$ cations, and an antiferromagnetic ordering of spins, which was found to be the ground state of a similar system (i.e. MIL-47$\mathrm{V}^{38}$ ), was considered for $\mathrm{Fe}^{3+}$. This recipe has been used successfully to predict the band gaps and electronic properties of hematite (i.e. $\left.\mathrm{Fe}_{2} \mathrm{O}_{3}\right)^{39}$ and also in MOFs. ${ }^{40-41}$ We find that the $\mathrm{D} 3$ scheme in conjunction with the HSE06 functional yield accurate lattice parameters and density of the narrow pore form of MIL-53-Al, in particular, which are stabilised by dispersion. ${ }^{22-23}$ The structural optimizations were considered converged if the maximum force on all atoms falls below $2.234 \mathrm{~kJ} \mathrm{~mol}^{-1} \AA^{-1}\left(4.5 \times 10^{-4} \mathrm{Hartree} \mathrm{Bohr}^{-1}\right)$. Calculations of MIL-53-Fe with different guest molecules were performed with a $1 \times 2 \times 1$ or $1 \times 2 \times 2$ expansion of the unit cell for guest molecules that have the same or different configurations in different channels. The initial structures that were optimized were taken from the observed crystal structures for different guest molecules in MIL-53-Fe. ${ }^{11,17,20}$ All calculations were performed at $0 \mathrm{~K}$, i.e. excluding any zero-point or thermal corrections to the energetics and structures.

\section{Results}

\subsection{Adsorption of quinone}

We first consider adsorption of quinone molecules in MIL-53-Fe. Combarieu et al. showed that experimentally, that MIL-53-Fe, when fully loaded with one quinone molecule per Fe center, adopt a parallel arrangement (see Figure 3a) at room temperature and transform to a "flip-flop" arrangement (see Figure 3b) upon heating to $140{ }^{\circ} \mathrm{C} .{ }^{20} \mathrm{We}$ have modelled these two configurations and calculated their relative stabilities and unit cell volumes, which are listed in Table 2. The main difference between configurations 1 and 2 is that in configuration 2, quinone molecules in half of the channels become orthogonal to quinone molecules in the other half of the channels, i.e. these quinone molecules are roughly parallel to the organic linkers of MIL-53Fe along lattice vector b' (note that the axes a'/b'/c', see inset of Figure 3, denote a rotated axes system with respect to the original cell axes, a rotation anticlockwise by $\sim 45^{\circ}$ around lattice parameter $\mathrm{c}$ ), while all quinone molecules in configuration 1 are roughly parallel to the organic linker of MIL-53-Fe along lattice vector a', see Figure $3 \mathrm{~b}$. Indeed, we find that configuration 1 is electronically more stable than configuration 2 by 2.18 $\mathrm{kJ} \mathrm{mol}^{-1}$ per quinone molecule. This is further evidenced by the relative stability of configuration 3 (see Figure $3 c$ ), in which all quinone molecules are parallel to the organic linker of MIL-53-Fe along lattice vector b', and we find that configuration 3 is less stable than configuration 1 by $3.43 \mathrm{~kJ} \mathrm{~mol}^{-1}$ per quinone molecule. Another minor difference between configurations 1 and 2 is that while the $\mathrm{O} \cdots \mathrm{O}$ axis of quinone molecules in configuration 1 are almost orthogonal to each other in the same channel, quinone molecules in the same channel in configuration 2 have the same orientations. Therefore, we consider a fourth adsorption configuration, configuration 4, see Figure 3d, and the difference between configurations 1 and 4, i.e. different molecular 
orientations in the channel, is highlighted by red arrows. We find that the relative stabilities of configurations 1 and 4 are almost identical, with configuration 4 being less stable than configuration 1 by only $0.07 \mathrm{~kJ} \mathrm{~mol}^{-1}$ per quinone molecule. At $300 \mathrm{~K}, k T$ is of the order of $2.5 \mathrm{~kJ} \mathrm{~mol}^{-1}$, and hence we expect that the quinone guests have sufficient thermal energy to transform between configurations 1 and 4. Pleasingly, both configurations 1 and 4 are seen in the experiment, evidenced from XRD. ${ }^{20}$ At $413 \mathrm{~K}, k T$ is of the order of $3.4 \mathrm{~kJ} \mathrm{~mol}^{-1}$, which exceeds the $2.2 \mathrm{~kJ} \mathrm{~mol}^{-1}$ energy difference between configurations 1 and 2 and hence this explains the transition to configuration 2 , as seen experimentally.

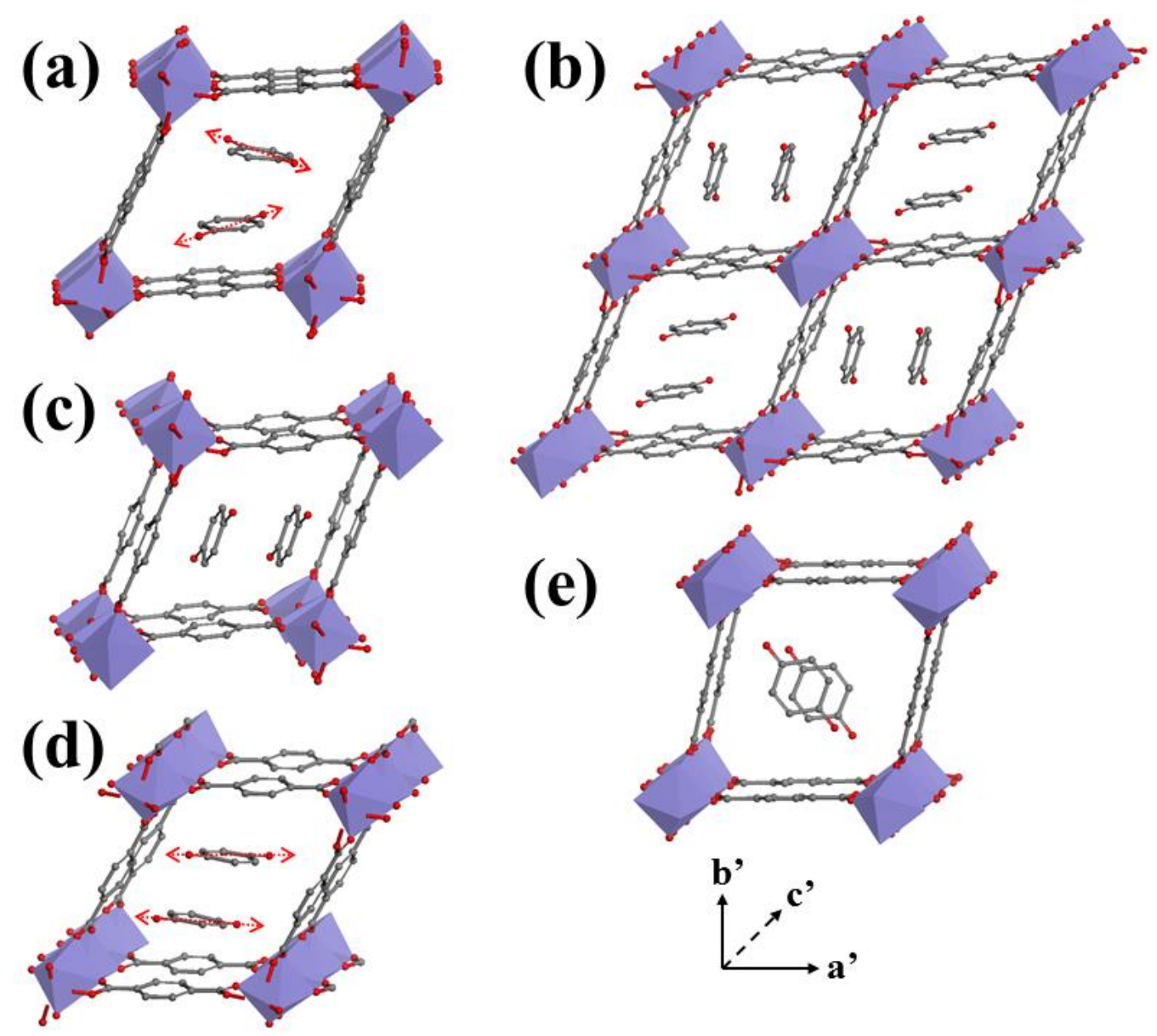

Figure 3: Adsorption configurations of quinone in MIL-53-Fe[quinone]: (a) configuration 1, (b) configuration 2, (c) configuration 3, (d) configuration 4, and (e) configuration 5. Hydrogen atoms and half of the guest molecules along the channel are omitted for clarity. Red: oxygen; grey: carbon; mauve: ironcentered octahedra.

\begin{tabular}{ccc}
\hline Configuration & $\Delta \mathrm{E}\left(\mathrm{kJ} \mathrm{mol}^{-1}\right.$ per guest $)$ & $\operatorname{Vol}\left(\AA^{3}\right)$ \\
\hline 1 & 0.00 & $1473.0\left(1455.3^{20}\right)$ \\
2 & 2.18 & $1483.5\left(1494.3^{20}\right)$ \\
3 & 3.41 & 1481.2 \\
4 & 0.07 & $1463.1\left(1455.3^{20}\right)$ \\
5 & 17.20 & 1578.1 \\
\hline
\end{tabular}

Table 2: Relative stabilities $(\Delta \mathrm{E})$ and unit cell volumes $(\mathrm{Vol})$ of different adsorption configurations considered for quinone in MIL-53-Fe. Experimental unit cell volumes are indicated in parentheses where known.

In addition to these four adsorption configurations, in which quinone molecules in the channel are parallel to the organic linker of MIL-53-Fe, either along lattice vector a' or along lattice vector b', we consider a fifth adsorption configuration (configuration 5, see Figure 3e), in which none of the quinone molecules in the channel is parallel to the organic linker of MIL-53-Fe. Instead, the quinone molecules are orthogonal to lattice vector c', and they are arranged in a way to maximize the $\mathrm{O}(\mathbf{G}) \cdots \mathrm{HO}(\mathbf{H})$ hydrogen bonding between 
quinone molecules and MIL-53-Fe. We find that configuration 5 is very unstable, being less stable than configuration 1 by $17.2 \mathrm{~kJ} \mathrm{~mol}^{-1}$ per quinone molecule. This demonstrates that the $\pi(\mathbf{G})-\pi(\mathbf{H})$ interaction is more favourable than hydrogen bonding between quinone molecules and MIL-53-Fe, and thermal transition to configuration 5 is unlikely to occur due to the $\sim 15.0 \mathrm{~kJ} \mathrm{~mol}^{-1}$ penalty of transforming from configuration 2 .

We note that the predicted volumes, which neglect thermal artefacts, are actually in good accord with experiment. Given that experiment sees both configuration 1 and 4 at room temperature, a fairer comparison of the predicted volume is a combination of the densities predicted for 1 and 4, giving an average room temperature volume of $1468 \AA^{3}$, which is slightly larger (i.e. less dense) than what is observed by experiment $1455.3 \AA^{3}$. The other experimental data point for volume is for configuration 2 , which is slightly underestimated by $\sim 11 \AA^{3}$ by theory. However, the overall error in volume is less than $1 \%$, lending further credence to the energies reported.

\subsection{Adsorption of pyridine}

Next, we consider adsorption of pyridine molecules in MIL-53-Fe. We start from the adsorption configuration (configuration 1) which was observed by experiment, ${ }^{11,}{ }^{17}$ see Figure $4 \mathrm{a}$, in which pairs of pyridine molecules are arranged head to tail to maximize the $\mathrm{N}(\mathbf{G}) \cdots \mathrm{HO}(\mathbf{H})$ hydrogen bonding between pyridine molecules and MIL-53-Fe. The distance between nitrogen donor of pyridine and oxygen in the hydroxide of MIL-53-Fe in the fully relaxed structure is $2.67 \AA$, and the distance between neighbouring pyridine molecules is $3.46 \AA$. Both structural parameters are in excellent agreement with experiment, which are 2.68 and $3.47 \AA$, respectively. ${ }^{17}$ While the $\mathrm{N}(\mathbf{G}) \cdots \mathrm{HO}(\mathbf{H})$ hydrogen bonding only involves nitrogen atoms of pyridine molecules, it would be interesting to see whether the internal rotations of pyridine molecules along the head-to-tail direction (i.e. lattice vector $\mathrm{b}$ in Figure 2a) affect the relative stability. So we constructed an additional configuration, 2 , see Figure $4 \mathrm{c}$, in which the pyridine molecules are rotated by approximately $40^{\circ}$ along the head-to-tail direction, compared with configuration 1 after full cell optimization. It turns out that configuration 2 is less stable than configuration 1 by only $2.21 \mathrm{~kJ} \mathrm{~mol}^{-1}$ per pyridine molecule, and the unit cell volumes are very similar as can be seen in Table 3. The $\mathrm{N}(\mathbf{G}) \cdots \mathrm{O}(\mathbf{H})$ distance and the distance between neighbouring pyridine molecules in configuration 2 are 2.68 and $3.45 \AA$, respectively, both of which are similar to those of configuration 1 and observed experimental values.

The relative stabilities of configurations 1 and 2 are quite similar for quinone and pyridine molecules in MIL-53-Fe, and a thermal transition is seen for quinone-loaded MIL-53-Fe at $140{ }^{\circ} \mathrm{C}$. Hence, it is reasonable to speculate that thermal transition to configuration 2 might occur at similar temperature for pyridine, although this might be more difficult to solve in experiment due to the high structural similarity of the two configurations. Additional rotamers were considered but these converged to configurations 1 or 2 , suggesting 2 is a minimum energy structure and not a transition state. Nevertheless, we cannot exclude the existence of other possible configurations with similar relative stabilities. We note that for pyridine, our theory appears to slightly overbind the guests, as evidenced by a slightly higher density. The relatively low energy of configuration 2 suggests that configuration 1 may be a structurally averaged picture, with the pyridine rattling in about their positions between configurations 1 and 2 . We presume that such rattling would lead to expansion of the crystal giving rise to an even closer agreement with the room temperature volume deduced from experiment.
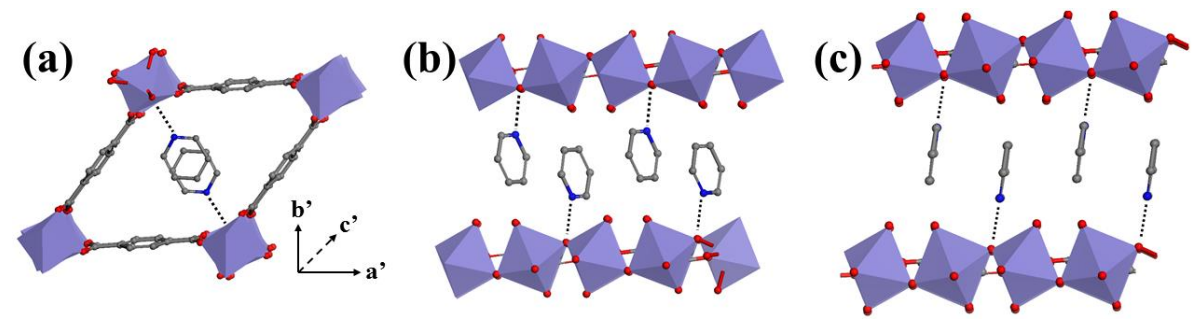

Figure 4: Adsorption configurations of pyridine in MIL-53-Fe[pyridine]: (a) configuration 1, view in the $a^{\prime} b$ ' plane (i.e. along the channel), (b) configuration 1, view in the $b^{\prime} c^{\prime}$ ' plane, and (c) configuration 2, view in the $b^{\prime} c^{\prime}$ ' plane. Hydrogen atoms are omitted for clarity, and hydrogen bonds are illustrated by black dotted lines. Red: oxygen; grey: carbon; blue: nitrogen; mauve: iron-centered octahedra. 


\begin{tabular}{ccc}
\hline Configuration & $\Delta \mathrm{E}\left(\mathrm{kJ} \mathrm{mol}^{-1}\right.$ per guest $)$ & $\mathrm{Vol}\left(\AA^{3}\right)$ \\
\hline 1 & 0.00 & $1376.6\left(1397.9^{17}\right)$ \\
2 & 2.21 & 1383.4 \\
\hline
\end{tabular}

Table 3: Relative stabilities $(\Delta \mathrm{E})$ and unit cell volumes $(\mathrm{Vol})$ of different adsorption configurations considered for pyridine in MIL-53-Fe[pyridine]. Experimental unit cell volumes are indicated in parentheses where known.

\subsection{Adsorption of 2,6-lutidine}

Finally, we look at adsorption of 2,6-lutidine in MIL-53-Fe. The presence of methyl groups in 2,6lutidine, prevents direct hydrogen bonding between nitrogen of 2,6-lutidine and the hydroxide of MIL-53-Fe because of unfavourable steric interactions between methyl groups of 2,6-lutidine and organic linkers of MIL53-Fe. It was therefore concluded that 2,6-lutidine molecules must co-adsorb with water molecules in the initial stage when 2,6-lutidine is introduced into hydrated MIL-53-Fe. ${ }^{17}$ The water molecules bridge 2,6-lutidine with MIL-53-Fe through two hydrogen bonds, one between the hydroxide of MIL-53-Fe and water, and another between water and the nitrogen atom of 2,6-lutidine. To get a qualitative picture about the competition of these different interactions, we constructed a configuration (1) of 2,6-lutidine in MIL-53-Fe without the presence of water, by replacing two of the hydrogens in the most stable configuration of pyridine in MIL-53-Fe with methyl groups, see Figure 5a. After full cell optimization, the unit cell volume of configuration 1 turns out to be 1458.9 $\AA^{3}$, somewhat smaller than the observed volume of $1593.2 \AA^{3}$ in experiment, ${ }^{17}$ which tends to suggest that this is not the phase observed in experiment. The distance between nitrogen of 2,6-lutidine and oxygen in the hydroxide of MIL-53-Fe is $2.99 \AA$, longer than that in pyridine by about $0.3 \AA$, which significantly weakens the $\mathrm{N}(\mathbf{G}) \cdots \mathrm{HO}(\mathbf{H})$ hydrogen bonding interaction. On the other hand, the distance between neighbouring 2,6lutidine molecules is $3.52 \AA$, which is similar to that of pyridine. We previously found two configurations for pyridine in MIL-53-Fe which are related by a simple rotation of pyridine molecules with relatively small energy cost. Adopting the same strategy for 2,6-lutidine, 2,6-lutidine molecules are rotated along the head-totail direction by about $30^{\circ}$, and we obtain another local minimum, configuration 2 , which is shown in Figure $5 \mathrm{c}$, and which is metastable to configuration 1 by only $0.32 \mathrm{~kJ} \mathrm{~mol}^{-1}$ per 2,6-lutidine molecule. The unit cell volume of configuration 2 is $1455.6 \AA^{3}$, very similar to that of configuration 1 . We also considered several other configurations of 2,6-lutidine similar to configurations 1, 3 and 4 of quinone, in which the 2,6-lutidine molecules were arranged roughly parallel of the organic linkers of MIL-53-Fe in order to maximize the $\pi(\mathbf{G})$ $\pi(\mathbf{H})$ interactions. These calculations result in configurations which are less stable than configuration 1 by more than $10 \mathrm{~kJ} \mathrm{~mol}^{-1}$ per 2,6-lutidine molecule, which indicates $\mathrm{N}(\mathbf{G}) \cdots \mathrm{HO}(\mathbf{H})$ hydrogen bonding is more favourable than $\pi(\mathbf{G})-\pi(\mathbf{H})$ interactions in the case of 2,6-lutidine.
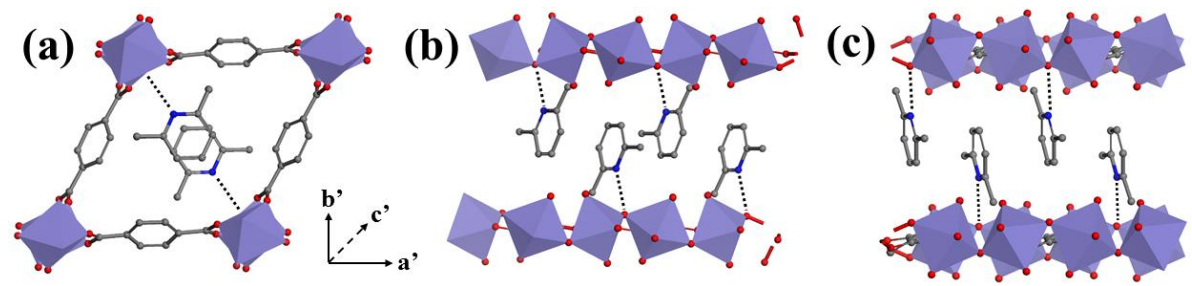

Figure 5: Adsorption configurations of 2,6-lutidine in MIL-53-Fe[2,6-lutidine]: (a) configuration 1, view in the $a$ ' $b$ ' plane (i.e. along the channel), (b) configuration 1, view in the $b^{\prime} c^{\prime}$ ' plane, and (c) configuration 2, view in the $b^{\prime} c$ ' plane. Hydrogen atoms are omitted for clarity, and hydrogen bonds are illustrated by black dotted lines. Red: oxygen; grey: carbon; blue: nitrogen; mauve: iron-centered octahedra.

We note in the experiment by Millange et al. ${ }^{17}$ upon heating of MIL-53-Fe[2,6-lutidine $+\mathrm{H}_{2} \mathrm{O}$ ], the material decomposed in a multi-step manner, first losing occluded water, and then losing various amounts of 2,6-lutidine molecules. During the dehydration process, Millange et al. were able to solve the crystal structure of an intermediate phase, MIL-53-Fe[2,6-lutidine 0.5$]$, corresponding to one 2,6-lutidine molecule per two $\mathrm{Fe}$ centers. Motivated by this observation, we consider two adsorption configurations of MIL-53-Fe[2,6lutidine $_{0.5}$ ], configurations 1 and 2 shown in Figures $6 \mathrm{~b}$ and $6 \mathrm{c}$ respectively. The primary difference between configurations 1 and 2 is that, in configuration 1 (Figure 6b), the two 2,6-lutidine molecules are arranged headto-tail, and nitrogen atoms of the two guest molecules form hydrogen bonds with $\mathrm{OH}^{-}$groups belong to two 
different inorganic chains, while in configuration 2 (Figure 6c), the two 2,6-lutidine molecules point towards the same direction, and nitrogen atoms of the two guest molecules form hydrogen bonds with $\mathrm{OH}^{-}$groups in the same inorganic chain. The relative stabilities and unit cell volumes of the two configurations are shown in Table 4. Configuration 1 is more stable than configuration 2 by about $2.62 \mathrm{~kJ} \mathrm{~mol}^{-1}$ per 2,6 -lutidine molecule, and the unit cell volumes are quite similar and close to the experiment value. Interestingly, the atomic positions of 2,6-lutidine molecules in MIL-53-Fe[2,6-lutidine ${ }_{0.5}$ ] as determined by experiment are fully disordered, which may be explained by the fact that configurations 1 and 2 are dynamically interchangeable in the channel due to the small energy difference between these configurations.
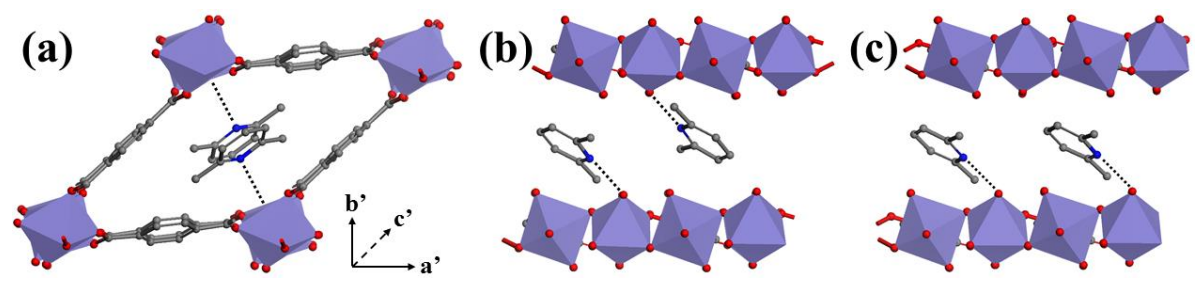

Figure 6: Adsorption configurations of 2,6-lutidine in MIL-53-Fe[2,6-lutidine ${ }_{0.5}$ ]: (a) configuration 1, view in the $a^{\prime} b$ ' plane (i.e. along the channel), (b) configuration 1, view in the $b^{\prime} c$ ' plane, and (c) configuration 2, view in the $b^{\prime} c$ ' plane. Hydrogen atoms are omitted for clarity, and hydrogen bonds are illustrated by black

dotted lines. Red: oxygen; grey: carbon; blue: nitrogen; mauve: iron-centered octahedra.

\begin{tabular}{ccc}
\hline Configuration & $\Delta \mathrm{E}\left(\mathrm{kJ} \mathrm{mol}^{-1}\right.$ per guest $)$ & $\mathrm{Vol}\left(\AA^{3}\right)$ \\
\hline 1 & 0.00 & $1188.7\left(1213.1^{17}\right)$ \\
2 & 2.62 & 1189.1
\end{tabular}

Table 4: Relative stabilities $(\Delta \mathrm{E})$ and unit cell volumes $(\mathrm{Vol})$ of different adsorption configurations considered for 2,6-lutidine in MIL-53-Fe[2,6-lutidine ${ }_{0.5}$ ]. Experimental unit cell volumes are indicated in parentheses where known.

\section{Discussions}

From the observations and inferences above, it is shown that due to the presence of multiple types of host-guest and guest-guest interactions which have comparable strengths (including $\pi-\pi, \mathrm{CH}-\pi$ and hydrogen bonds), the competition between these different interactions changes with temperature and the result is that MIL-53-Fe can support multiple different configurations of a particular guest. In some instances, a transition between configurations is calculated to incur a very modest energy penalty, and for quinone, these small energy differences are manifested by evidence of orientational disorder, suggesting dynamic switching between configurations. The MIL-53 material has an innate flexibility which probably helps to lower the transition barrier between different possible configurations of guests even at room temperature. We suspect this could be one of the reasons why unique geometries have not been identified for several of the organic molecules which have been adsorbed in MIL-53-Fe, such as para-xylene. ${ }^{11}$ We also note that in our simulation, we consider cases in which MIL-53-Fe is fully loaded with guest molecules, i.e. one guest molecule per Fe center for computational expediency. In various experiments of adsorption in MIL-53-Fe, the loading of guests inside the pore is usually less than 1 per Fe, e.g. it has been shown that the maximum loading of pyridine in MIL-53$\mathrm{Fe}$ is 0.85 pyridine molecule per Fe. ${ }^{19}$ Because the channels are not fully occupied by pyridine molecules, neighbouring pyridine molecules in the vicinity of these "vacancies" have higher configurational entropy since they are less sterically constrained by neighbours. This provides another source of disordering, which makes the adsorption geometry more difficult to resolve.

To understand the difference between adsorption of pyridine and 2,6-lutidine in MIL-53-Fe, we calculated the binding energies between the host and guest molecules, at the most stable configurations, either resolved by experiments or identified by our simulations. As mentioned earlier, pyridine molecules displace water molecules in MIL-53-Fe[ $\left.\mathrm{H}_{2} \mathrm{O}\right]$, while 2,6-lutidine molecules do not displace water molecules in MIL$53-\mathrm{Fe}\left[\mathrm{H}_{2} \mathrm{O}\right]$. Instead, the water molecules remain in place and bridge 2,6-lutidine molecules with MIL-53-Fe. To examine this, we constructed configurations of pyridine and 2,6-lutidine adsorbed in MIL-53-Fe with water molecules, based on the experimentally deduced structure of MIL-53-Fe[2,6-lutidine $\left.+\mathrm{H}_{2} \mathrm{O}\right] \cdot{ }^{17}$ We note here, for MIL-53-Fe[pyridine $+\mathrm{H}_{2} \mathrm{O}$ ] and MIL-53-Fe[2,6-lutidine $+\mathrm{H}_{2} \mathrm{O}$ ], the fully occupied host actually has two 
guest molecules per Fe center, i.e. one water plus one 2,6-lutidine or one pyridine. The calculated binding energies and unit cell volumes are summarized in Table 5. For MIL-53-Fe[pyridine $+\mathrm{H}_{2} \mathrm{O}$ ] and MIL-53$\mathrm{Fe}\left[2\right.$,6-lutidine $+\mathrm{H}_{2} \mathrm{O}$ ], the binding energies were calculated between pyridine/2,6-lutidine molecules and MIL-53-Fe[ $\left.\mathrm{H}_{2} \mathrm{O}\right]$, i.e. water is regarded as part of the host. The unit cell volumes of MIL-53-Fe[pyridine + $\mathrm{H}_{2} \mathrm{O}$ ] and MIL-53-Fe[2,6-lutidine $+\mathrm{H}_{2} \mathrm{O}$ ] are quite similar, and this is most likely due to effects of coadsorption of water molecules, causing MIL-53-Fe to be fully expanded.

Two sets of the binding energies were calculated, one set contains the guest-guest interaction, and in the other set, the guest-guest interactions were subtracted out. The guest-guest interactions were calculated for a cell with only two guest molecules at fixed geometry in fully loaded MIL-53-Fe or MIL-53-Fe[ $\left.\mathrm{H}_{2} \mathrm{O}\right]$. If we look at the second set of binding energies, we find that the binding of pyridine in MIL-53-Fe[pyridine] is stronger than that of water and 2,6-lutidine by $25 \sim 29 \mathrm{~kJ} \mathrm{~mol}^{-1}$ per guest molecule, and this provides the thermodynamic driving force for the displacement of water molecules in MIL-53- $\mathrm{Fe}\left[\mathrm{H}_{2} \mathrm{O}\right]$ upon exposure to pyridine. For 2,6-lutidine, the binding energy in MIL-53-Fe[2,6-lutidine] is more similar to that of water, and this hints at why 2,6-lutidine does not displace all the water in MIL-53-Fe[ $\left.\mathrm{H}_{2} \mathrm{O}\right]$. However, upon heating and the loss of half the 2,6-lutidine guest molecules, the binding energy of 2,6-lutidine in MIL-53-Fe[2,6-lutidine ${ }_{0.5}$ ] is much larger; an increase from -77.4 to $-104.8 \mathrm{~kJ} \mathrm{~mol}^{-1}$ per 2,6-lutidine molecule. We find that the host is stabilized by the dispersion interactions between organic linkers in the half-open form of MIL-53-Fe[2,6lutidine ${ }_{0.5}$, by about $11.5 \mathrm{~kJ} \mathrm{~mol}^{-1}$ per Fe, compared with that in the open form of MIL-53-Fe[2,6-lutidine]. The host-guest interaction in MIL-53-Fe[2,6-lutidine ${ }_{0.5}$ ] is also strengthened due to: (1) decreasing steric repulsion interactions between methyl groups of 2,6-lutidine and organic linkers of MIL-53-Fe, and (2) increasing $\pi-\pi$ interactions between 2,6-lutidine and organic linkers of MIL-53-Fe. Although the guest-guest interaction in MIL-53-Fe[2,6-lutidine ${ }_{0.5}$ ] is weakened, it represents a relatively small energetic contribution compared with the enhancement in host-host and host-guest interactions. In addition, the relative stabilities between the two most stable adsorption configurations in MIL-53-Fe[2,6-lutidine] and MIL-53-Fe[2,6lutidine $\left._{0.5}\right]$ are 0.32 and $2.62 \mathrm{~kJ} \mathrm{~mol}^{-1}$ per 2,6-lutidine molecule, respectively. This suggests that guest configurations in MIL-53-Fe[2,6-lutidine] are more likely to be disordered than those in MIL-53-Fe[2,6lutidine $_{0.5}$. This could explain why the crystal structure of MIL-53-Fe[2,6-lutidine ${ }_{0.5}$ ] could be solved whilst MIL-53-Fe[2,6-lutidine] could not be isolated. ${ }^{17}$

Next, we look at the binding energies of pyridine and 2,6-lutidine in MIL-53- $\mathrm{Fe}\left[\mathrm{H}_{2} \mathrm{O}\right]$, see Figure 7 , and we can find that the effect of the presence of water in the adsorption of 2,6-lutidine is significant. The binding energy of 2,6-lutidine in MIL-53- $\mathrm{Fe}\left[\mathrm{H}_{2} \mathrm{O}\right]$ is larger than that in anhydrous MIL-53-Fe by $32.2 \mathrm{~kJ} \mathrm{~mol}^{-}$ ${ }^{1}$ per 2,6-lutidine molecule ignoring guest-guest interactions and $31.5 \mathrm{~kJ} \mathrm{~mol}^{-1}$ if guest-guest interactions are included. Obviously, this is due to the strong hydrogen bonds between water and nitrogen donors of 2,6lutidine, and this explains why 2,6-lutidine molecules do not displace water molecules in MIL-53-Fe[ $\left.\mathrm{H}_{2} \mathrm{O}\right]$. On the other hand, the binding energy of pyridine in MIL-53-Fe $\left[\mathrm{H}_{2} \mathrm{O}\right]$ hardly changes with the presence of water, implying the hydrogen bonding between pyridine and water and that between pyridine and MIL-53-Fe are similar in strength. At the same time, we find that the binding energy of pyridine in MIL-53- $\mathrm{Fe}\left[\mathrm{H}_{2} \mathrm{O}\right]$ is weaker than that of 2,6-lutidine in $\mathrm{MIL}-53-\mathrm{Fe}\left[\mathrm{H}_{2} \mathrm{O}\right]$ by about $2.1 \mathrm{~kJ} \mathrm{~mol}^{-1}$ per guest molecule. This is partly due to the additional $\mathrm{CH}_{3}(\mathbf{G})-\pi(\mathbf{H})$ interactions between methyl groups of 2,6-lutidine and organic linkers of MIL-53-Fe, see Figure 7b. In addition, because of the water molecules between pyridine and MIL-53-Fe, the relative shift between the "head" and the "tail" of two neighbouring pyridine molecules increases from $0.96 \AA$ to $1.16 \AA$, i.e. there is less overlap between neighbouring pyridine molecules, and it weakens the $\pi-\pi$ interactions between neighbouring pyridine molecules, from $-12.6 \mathrm{~kJ} \mathrm{~mol}^{-1}$ per pyridine molecule in MIL-53Fe to $-9.6 \mathrm{~kJ} \mathrm{~mol}^{-1}$ per pyridine molecule in MIL-53-Fe[ $\left.\mathrm{H}_{2} \mathrm{O}\right]$. With respect to 2,6-lutidine, the $\pi-\pi$ interactions between neighbouring 2,6-lutidine molecules are only weakened by less than $0.4 \mathrm{~kJ} \mathrm{~mol}^{-1}$ per 2,6-lutidine molecule, from $-18.5 \mathrm{~kJ} \mathrm{~mol}^{-1}$ per 2,6-lutidine molecule in MIL-53-Fe to $-18.1 \mathrm{~kJ} \mathrm{~mol}^{-1}$ per 2,6-lutidine molecule in MIL-53-Fe[ $\left.\mathrm{H}_{2} \mathrm{O}\right]$. Comparing the binding energies which include guest-guest interactions, we find adsorption of 2,6-lutidine in MIL-53-Fe[H $\left.\mathrm{H}_{2} \mathrm{O}\right]$ is more favourable than pyridine by $19.1 \mathrm{~kJ} \mathrm{~mol}^{-1}$ per guest molecule, and we conclude this is due to stronger guest-guest interactions and additional $\mathrm{CH}_{3}(\mathbf{G})-\pi(\mathbf{H})$ interactions between methyl groups of 2,6-lutidine and organic linkers of MIL-53-Fe. This explains the experimentally observed selective adsorption of 2,6-lutidine over pyridine in MIL-53- $\mathrm{Fe}\left[\mathrm{H}_{2} \mathrm{O}\right] .{ }^{17}$ 

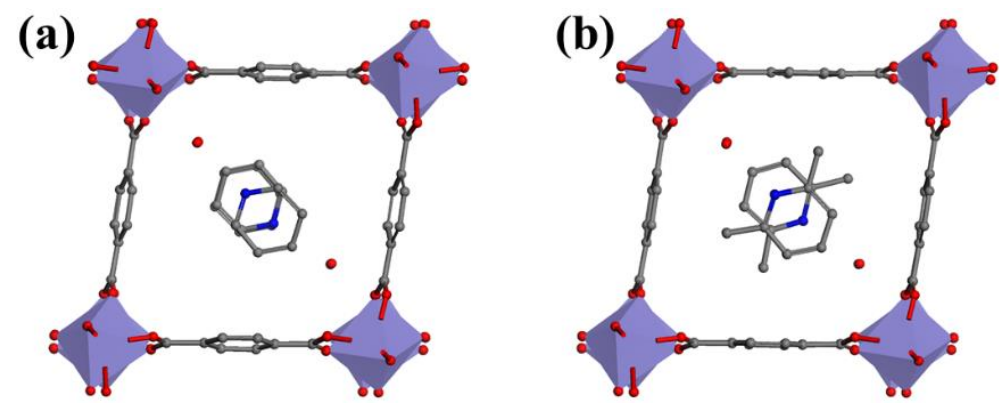

Figure 7: Adsorption configurations of (a) pyridine and (b) 2,6-lutidine in MIL-53-Fe[pyridine $+\mathrm{H}_{2} \mathrm{O}$ ] and MIL-53-Fe[2,6-lutidine $+\mathrm{H}_{2} \mathrm{O}$ ]. Hydrogen atoms are omitted for clarity. Red: oxygen; grey: carbon; blue: nitrogen; mauve: iron-centered octahedra.

\begin{tabular}{cccc}
\hline Guest & $\mathrm{E}_{\mathrm{b}}(\mathrm{w} / \mathrm{G}-\mathrm{G})$ & $\mathrm{E}_{\mathrm{b}}(\mathrm{w} / \mathrm{o} \mathrm{G}-\mathrm{G})$ & $\mathrm{Vol}\left(\AA^{3}\right)$ \\
\hline water & -62.0 & -36.7 & $973.1\left(1000.6^{11}\right)$ \\
pyridine & -91.2 & -65.9 & $1376.6\left(1397.9^{17}\right)$ \\
pyridine + water & -89.8 & -70.6 & 1576.4 \\
2,6-lutidine & -77.4 & -40.5 & 1458.9 \\
2,6-lutidine + water & -108.9 & -72.7 & $1579.1\left(1593.2^{17}\right)$ \\
2,6-lutidine 0.5 & -104.8 & -91.7 & $1188.7\left(1213.1^{17}\right)$ \\
\hline
\end{tabular}

Table 5: Binding energies (in $\mathrm{kJ} \mathrm{mol}^{-1}$ per guest molecule) and unit cell volumes (Vol, in $\AA^{3}$ ) of different guests in MIL-53-Fe. $E_{b}(w / G-G)$ and $E_{b}$ (w/o G-G) indicate binding energies with and without guest-guest interactions, respectively. Experimental unit cell volumes are indicated in the brackets.

\section{Conclusions}

In summary, we have studied the adsorptions of 1,4-benzoquinone, pyridine and 2,6-lutidine molecules, and the co-adsorptions of water and pyridine or 2,6-lutidine molecules, in MIL-53-Fe. We find that due to the competitions of various host-guest and guest-guest interactions which have similar strengths, it is likely that these guests can easily overcome modest thermal barriers to visit several different configurations at room temperature over a rapid timescale, which is manifested as conformational disorder when the structures are studied using diffraction methods. We expect similar behaviours for other organic molecules in MIL-53Fe. Our study provides an explanation for the discrepancy between the adsorption behaviours of two organic molecules that are structurally similar, pyridine and 2,6-lutidine in MIL-53-Fe. The selective adsorption of pyridine and 2,6-lutidine in MIL-53-Fe and MIL-53-Fe[ $\left.\mathrm{H}_{2} \mathrm{O}\right]$ results from a subtle balance of different types of host-guest and guest-guest interactions, which can be modulated by the presence of water molecules as well as functionalization of the guest molecules. We note the energy differences between the multiple adsorption configurations considered for different guest molecules in MIL-53-Fe are small enough that the population of a particular guest adsorption configuration will likely be influenced by entropic in addition to enthalpic contributions at a given temperature and pressure. We conclude that in addition to molecular size, ${ }^{11,18}$ the detailed host-guest and guest-guest interactions are required to understand observed separation behaviours and that simulation approaches can discern the delicate balance of forces and offers an additional means of designing and screening materials for potential applications using MOFs.

\section{Acknowledgements}

SL and BS acknowledge financial support from EPSRC (EP/K039296/1). Through our membership of the UK's HPC Materials Chemistry Consortium, which is funded by EPSRC (EP/L000202), this work made use of the facilities of HECToR and ARCHER, the UK's national high-performance computing service, which is funded by the Office of Science and Technology through EPSRC's High End Computing Programme.

\section{References}

1. Peng, Y.; Li, Y.; Ban, Y.; Jin, H.; Jiao, W.; Liu, X.; Yang, W., Membranes. Metal-organic framework nanosheets as building blocks for molecular sieving membranes. Science 2014, 346 (6215), 1356-9. 
2. Murray, L. J.; Dinca, M.; Long, J. R., Hydrogen storage in metal-organic frameworks. Chemical Society Reviews 2009, 38 (5), 1294-314.

3. Millward, A. R.; Yaghi, O. M., Metal-organic frameworks with exceptionally high capacity for storage of carbon dioxide at room temperature. Journal of the American Chemical Society 2005, 127 (51), 17998-9.

4. Mason, J. A.; Veenstra, M.; Long, J. R., Evaluating metal-organic frameworks for natural gas storage. Chemical Science 2014, 5 (1), 32.

5. Kreno, L. E.; Leong, K.; Farha, O. K.; Allendorf, M.; Van Duyne, R. P.; Hupp, J. T., Metal-organic framework materials as chemical sensors. Chemical Reviews 2012, 112 (2), 1105-25.

6. Horcajada, P.; Serre, C.; Vallet-Regí, M.; Sebban, M.; Taulelle, F.; Férey, G., Metal-Organic Frameworks as Efficient Materials for Drug Delivery. Angewandte Chemie 2006, 118 (36), 6120-6124.

7. Férey, G.; Millange, F.; Morcrette, M.; Serre, C.; Doublet, M. L.; Grenèche, J. M.; Tarascon, J. M., Mixed-valence li/fe-based metal-organic frameworks with both reversible redox and sorption properties. Angewandte Chemie International Edition 2007, 46 (18), 3259-63.

8. $\quad$ Lee, J.; Farha, O. K.; Roberts, J.; Scheidt, K. A.; Nguyen, S. T.; Hupp, J. T., Metal-organic framework materials as catalysts. Chemical Society Reviews 2009, 38 (5), 1450-9.

9. $\quad$ Furukawa, H.; Müller, U.; Yaghi, O. M., "Heterogeneity within Order” in Metal-Organic Frameworks. Angewandte Chemie International Edition 2015, DOI: 10.1002/anie.201410252.

10. Tranchemontagne, D. J.; Mendoza-Cortés, J. L.; O'Keeffe, M.; Yaghi, O. M., Secondary building units, nets and bonding in the chemistry of metal-organic frameworks. Chemical Society Reviews 2009, 38 (5), 125783.

11. Millange, F.; Serre, C.; Guillou, N.; Férey, G.; Walton, R. I., Structural effects of solvents on the breathing of metal-organic frameworks: an in situ diffraction study. Angewandte Chemie International Edition 2008, 47 (22), 4100-5.

12. Liu, Y.; Her, J.-H.; Dailly, A.; Ramirez-Cuesta, A. J.; Neumann, D. A.; Brown, C. M., Reversible structural transition in MIL-53 with large temperature hysteresis. Journal of the American Chemical Society 2008, 130 (35), 11813-8.

13. Drisdell, W. S.; Poloni, R.; McDonald, T. M.; Long, J. R.; Smit, B.; Neaton, J. B.; Prendergast, D.; Kortright, J. B., Probing adsorption interactions in metal-organic frameworks using X-ray spectroscopy. Journal of the American Chemical Society 2013, 135 (48), 18183-90.

14. Coudert, F. X.; Jeffroy, M.; Fuchs, A. H.; Boutin, A.; Mellot-Draznieks, C., Thermodynamics of guestinduced structural transitions in hybrid organic-inorganic frameworks. Journal of the American Chemical Society 2008, 130 (43), 14294-302.

15. Coudert, F.-X., Responsive Metal-Organic Frameworks and Framework Materials: Under Pressure, Taking the Heat, in the Spotlight, with Friends. Chemistry of Materials 2015, 27 (6), 1905-1916.

16. Serre, C.; Millange, F.; Thouvenot, C.; Noguès, M.; Marsolier, G.; Louër, D.; Férey, G., Very Large Breathing Effect in the First Nanoporous Chromium(III)-Based Solids: MIL-53 or Cr III (OH) $\{\mathrm{O} 2 \mathrm{C}-\mathrm{C} 6 \mathrm{H}$ $4-\mathrm{CO} 2\} \cdot\{\mathrm{HO} 2 \mathrm{C}-\mathrm{C} 6 \mathrm{H} 4-\mathrm{CO} 2 \mathrm{H}\} \times$ x $\cdot \mathrm{H} 2 \mathrm{O}$ y. Journal of the American Chemical Society 2002, 124 (45), 13519-13526.

17. Millange, F.; Guillou, N.; Medina, M. E.; Férey, G.; Carlin-Sinclair, A.; Golden, K. M.; Walton, R. I., Selective Sorption of Organic Molecules by the Flexible Porous Hybrid Metal-Organic Framework MIL53(Fe) Controlled by Various Host-Guest Interactions. Chemistry of Materials 2010, 22 (14), 4237-4245.

18. Férey, G.; Serre, C., Large breathing effects in three-dimensional porous hybrid matter: facts, analyses, rules and consequences. Chemical Society Reviews 2009, 38 (5), 1380-99.

19. Whitfield, T. R.; Wang, X.; Liu, L.; Jacobson, A. J., Metal-organic frameworks based on iron oxide octahedral chains connected by benzenedicarboxylate dianions. Solid State Sciences 2005, 7 (9), 1096-1103.

20. de Combarieu, G.; Morcrette, M.; Millange, F.; Guillou, N.; Cabana, J.; Grey, C. P.; Margiolaki, I.; Férey, G.; Tarascon, J. M., Influence of the Benzoquinone Sorption on the Structure and Electrochemical Performance of the MIL-53(Fe) Hybrid Porous Material in a Lithium-Ion Battery. Chemistry of Materials 2009, 21 (8), 1602-1611.

21. Klimeš, J.; Michaelides, A., Perspective: Advances and challenges in treating van der Waals dispersion forces in density functional theory. Journal of Chemical Physics 2012, 137 (12), 120901-120901.

22. Nanthamathee, C.; Ling, S.; Slater, B.; Attfield, M. P., Contradistinct Thermoresponsive Behavior of Isostructural MIL-53 Type Metal-Organic Frameworks by Modifying the Framework Inorganic Anion. Chemistry of Materials 2015, 27 (1), 85-95.

23. Walker, A. M.; Civalleri, B.; Slater, B.; Mellot-Draznieks, C.; Corà, F.; Zicovich-Wilson, C. M.; Román-Pérez, G.; Soler, J. M.; Gale, J. D., Flexibility in a metal-organic framework material controlled by 
weak dispersion forces: the bistability of MIL-53(Al). Angewandte Chemie International Edition 2010, 49 (41), 7501-3.

24. Ling, S.; Slater, B., Assessment of van der Waals Corrected Density Functional Theory Methods for Screening the Stability of Nanoporous Framework Materials. In preparation 2015.

25. Chen, L.; Mowat, J. P. S.; Fairen-Jimenez, D.; Morrison, C. A.; Thompson, S. P.; Wright, P. A.; Düren, T., Elucidating the breathing of the metal-organic framework MIL-53(Sc) with ab initio molecular dynamics simulations and in situ X-ray powder diffraction experiments. Journal of the American Chemical Society 2013, 135 (42), 15763-73.

26. Addicoat, M. A.; Vankova, N.; Akter, I. F.; Heine, T., Extension of the Universal Force Field to MetalOrganic Frameworks. Journal of Chemical Theory and Computation 2014, 10 (2), 880-891.

27. Kim, J.; Lin, L.-C.; Lee, K.; Neaton, J. B.; Smit, B., Efficient Determination of Accurate Force Fields for Porous Materials Using ab Initio Total Energy Calculations. Journal of Physical Chemistry C 2014, 118 (5), 2693-2701.

28. Fang, H.; Demir, H.; Kamakoti, P.; Sholl, D. S., Recent developments in first-principles force fields for molecules in nanoporous materials. Journal of Materials Chemistry A 2014, 2 (2), 274.

29. VandeVondele, J.; Krack, M.; Mohamed, F.; Parrinello, M.; Chassaing, T.; Hutter, J., Quickstep: Fast and accurate density functional calculations using a mixed Gaussian and plane waves approach. Computer Physics Communications 2005, 167 (2), 103-128.

30. Hutter, J.; Iannuzzi, M.; Schiffmann, F.; VandeVondele, J., cp2k: atomistic simulations of condensed matter systems. Wiley Interdisciplinary Reviews: Computational Molecular Science 2014, 4 (1), 15-25.

31. Goedecker, S.; Teter, M.; Hutter, J., Separable dual-space Gaussian pseudopotentials. Physical Review B 1996, 54 (3), 1703-1710.

32. Krack, M., Pseudopotentials for $\mathrm{H}$ to $\mathrm{Kr}$ optimized for gradient-corrected exchange-correlation functionals. Theoretical Chemistry Accounts 2005, 114 (1-3), 145-152.

33. Boys, S. F.; Bernardi, F., The calculation of small molecular interactions by the differences of separate total energies. Some procedures with reduced errors. Molecular Physics 1970, 19 (4), 553-566.

34. Krukau, A. V.; Vydrov, O. A.; Izmaylov, A. F.; Scuseria, G. E., Influence of the exchange screening parameter on the performance of screened hybrid functionals. Journal of Chemical Physics 2006, 125 (22), $224106-224106$.

35. Grimme, S.; Antony, J.; Ehrlich, S.; Krieg, H., A consistent and accurate ab initio parametrization of density functional dispersion correction (DFT-D) for the 94 elements H-Pu. Journal of Chemical Physics 2010, 132 (15), 154104-154104.

36. Axilrod, B. M.; Teller, E., Interaction of the van der Waals Type Between Three Atoms. Journal of Chemical Physics 1943, 11 (6), 299.

37. Guidon, M.; Hutter, J.; VandeVondele, J., Auxiliary Density Matrix Methods for Hartree-Fock Exchange Calculations. Journal of Chemical Theory and Computation 2010, 6 (8), 2348-2364.

38. Vanpoucke, D. E. P.; Jaeken, J. W.; De Baerdemacker, S.; Lejaeghere, K.; Van Speybroeck, V., Quasi1D physics in metal-organic frameworks: MIL-47(V) from first principles. Beilstein Journal of Nanotechnology 2014, 5 (1), 1738-1748.

39. Pozun, Z. D.; Henkelman, G., Hybrid density functional theory band structure engineering in hematite. Journal of Chemical Physics 2011, 134 (22), 224706-224706.

40. Hendon, C. H.; Tiana, D.; Fontecave, M.; Sanchez, C.; D'arras, L.; Sassoye, C.; Rozes, L.; MellotDraznieks, C.; Walsh, A., Engineering the Optical Response of the Titanium-MIL-125 Metal-Organic Framework through Ligand Functionalization. Journal of the American Chemical Society 2013, 135 (30), 10942-5.

41. Butler, K. T.; Hendon, C. H.; Walsh, A., Electronic chemical potentials of porous metal-organic frameworks. Journal of the American Chemical Society 2014, 136 (7), 2703-6. 